\title{
Classificação orientada a objetos aplicada na caracterização da cobertura da terra no Araguaia
}

\author{
Rener Ribeiro Fernandes ${ }^{(1)}$, Gustavo Manzon Nunes $^{(1)}$ e Thiago Sanna Freire Silva(2) \\ (1) Universidade Federal de Mato Grosso, Faculdade de Engenharia Florestal, Programa de Pós-Graduação em Ciências \\ Florestais e Ambientais, Avenida Fernando Correa da Costa, s/no, CEP 78060-900 Cuiabá, MT. E-mail: renerfernandes@florestal.eng.br, \\ gustavonunes@ufmt.br (2)Instituto Nacional de Pesquisas Espaciais, Divisão de Sensoriamento Remoto, Avenida dos Astronautas, no 1.758, \\ Jardim da Granja, CEP 12227-010 São José dos Campos, SP. E-mail: thiago@ltid.inpe.br
}

Resumo - O objetivo deste trabalho foi utilizar a classificação orientada a objetos em imagens TM/ Landsat-5, para caracterizar classes de uso e cobertura da terra, na região do Médio Araguaia. A cena 223/068, adquirida em 5/9/2010, foi submetida a correção radiométrica, atmosférica e geométrica, como etapas de pré-processamento. Em seguida, foram geradas duas imagens por meio das matemáticas de bandas espectrais do índice de vegetação por diferença normalizada (NDVI) e do índice de água por diferença normalizada modificado (MNDWI), utilizados na classificação de imagens. Para a segmentação destas, utilizaram-se os parâmetros de escala 250, 200, 150, 100, 50, os algoritmos "assign class" e "nearest neighbor", e os descritores de média, área e relação de borda. Foi empregada matriz de confusão, para avaliar a acurácia da classificação, por meio do coeficiente de exatidão global e do índice de concordância Kappa. A exatidão global para o mapeamento foi de 83,3\%, com coeficiente Kappa de 0,72. A classificação foi feita quanto às fitofisionomias do Cerrado, ao uso antrópico e urbano da terra, a corpos d'água e a bancos de areia. As matemáticas de bandas espectrais utilizadas apresentam resultados promissores no delineamento das classes de cobertura da terra no Araguaia.

Termos para indexação: classificação digital de imagens, fitofisionomias do Cerrado, MNDWI, NDVI, segmentação de imagens, sensoriamento remoto.

\section{Object-oriented classification applied to the characterization of soil use and land cover in the Araguaia, Brazil}

\begin{abstract}
The objective of this work was to use object-oriented classification in TM/Landsat-5 images to characterize land use and land cover classes in the Araguaia region. The scene 223/068, acquired on 9/5/2010, was subjected to the following pre-processing stages: radiometric, atmospheric, and geometric corrections. Two images were generated by the mathematical spectral bands normalized difference vegetation index (NDVI) and modified normalized difference water index (MNDWI), which were used in the classification process. For image segmentation, the scale parameters $250,200,150,100,50$, the algorithms assign class and nearest neighbor, and the attributes of average, area, and border ratio were used. A confusion matrix was used to assess the accuracy of the classification, using the overall accuracy coefficient and the Kappa index of agreement. Overall accuracy for mapping was $83.3 \%$, with Kappa coefficient of 0.72 . The classification was done as to Cerrado physiognomies, anthropic and urban use of the land, water bodies, and sand banks. The mathematical spectral bands used are promising for delineating classes of the land cover in Araguaia.
\end{abstract}

Index terms: digital image classification, Cerrado physiognomies, MNDWI, NDVI, image segmentation, remote sensing.

\section{Introdução}

A região do Araguaia e da Ilha do Bananal faz parte de um complexo mosaico de unidades inter-relacionadas que regula a mais importante área de ecossistemas aquáticos do Cerrado brasileiro. Estudos com informações confiáveis sobre a distribuição espacial do uso da terra, nessa região, ainda são escassos, apesar de fundamentais para direcionar o planejamento e a definição de prioridades por parte do poder público e privado. Assim, o desenvolvimento de metodologias que auxiliem no monitoramento e no ordenamento do uso da terra ainda é necessário.

Os avanços tecnológicos na área de sensoriamento remoto nos últimos anos têm possibilitado a aquisição de informações mais precisas e detalhadas. Um exemplo desse avanço é a classificação orientada a objetos geográficos, que é eficiente no mapeamento 
do uso da terra (Luz et al., 2010). Esta classificação baseia-se no agrupamento de pixels a partir da segmentação da imagem. Neste método, a segmentação é o passo preliminar que divide a imagem em objetos homogêneos e contíguos, e a acurácia da segmentação afeta diretamente o desempenho da classificação (Yan et al., 2006). Duveiller et al. (2008) relataram que melhorias recentes no processo de segmentação de imagens tornaram a classificação orientada a objetos importante ferramenta para delinear classes de uso e cobertura da terra de forma eficiente.

Em trabalho pioneiro com classificação orientada a objetos no Brasil, Antunes (2003) buscou mapear tipologias vegetais em ambiente ciliar do Rio Paracatu, no Paraná, a partir do uso de imagens Ikonos. O autor comparou os resultados obtidos por métodos tradicionais de classificação automática baseados em pixels, como máxima verossimilhança, lógica fuzzy e classificação orientada a objetos, e verificou a superioridade deste último. Nessa linha de estudo, destacam-se os trabalhos de Alves et al. (2009), Luz et al. (2010) e Pinho et al. (2012).

A classificação orientada a objetos pode ser utilizada em conjunto com outras técnicas de processamento digital de imagens, como as matemáticas de bandas espectrais índice de vegetação por diferença normalizada (NDVI) e índice de água por diferença normalizada modificado (MNDWI).

O NDVI, proposto por Rouse et al. (1973), tornou possível - por sua simplicidade e relativa alta sensibilidade à densidade da cobertura vegetal comparações espaciais e temporais da atividade fotossintética terrestre, bem como o monitoramento sazonal, interanual e de variações de longo prazo de parâmetros estruturais, fenológicos e biofísicos da vegetação em escala global (Wang et al., 2003). O MNDWI, proposto por Xu (2006), consiste em uma razão de expressão modificada do NDWI, desenvolvido por McFeeters (1996), para delineamento de feições relacionadas à água. A diferença entre os dois métodos é que o NDWI utiliza o canal do infravermelho próximo, enquanto o MNDWI considera o canal do infravermelho médio no processo de razão de bandas espectrais. Entretanto, o NDWI não separa, de forma eficiente, o solo da água, o que provoca certa mistura entre as respostas espectrais desses dois alvos. Quanto maior o contraste de água na imagem MNDWI, mais precisa será a extração de corpos d'água de áreas urbanas, solo exposto e vegetação. Neste caso, os corpos d'água apresentarão valores negativos, que podem ser desconsiderados (Xu, 2006).

Essas matemáticas de bandas espectrais podem ser inseridas como parâmetros de entrada no processo de classificação orientada a objetos, para ampliar o grau de informação para caracterização dos alvos no processo do levantamento de uso e cobertura da terra.

O objetivo deste trabalho foi utilizar a classificação orientada a objetos em imagens TM/Landsat-5, para caracterizar classes de uso e cobertura da terra, na região do Médio Araguaia.

\section{Material e Métodos}

A área de estudo compreende uma extensão de 194.054 ha, localizada na região do Médio Araguaia, entre o Município de São Félix do Araguaia, MT, e a Ilha

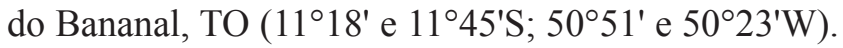
O relevo da região é plano em praticamente toda sua extensão, com pouquíssimos morros residuais isolados (Mileski, 1994). O clima, segundo a classificação de Köppen, é do tipo Aw, com temperaturas médias anuais de $24^{\circ} \mathrm{C}$. A vegetação é característica do bioma Cerrado e apresenta unidades fitofisionômicas típicas, como mata de galeria, cerrado típico, cerradão, varjão sujo, varjão limpo e ipucas.

As classes consideradas no mapeamento de cobertura da terra abrangeram as fitofisionomias do cerrado, o uso urbano e agrícola da terra, os cursos d'água e os bancos de areia. As fitofisionomias avaliadas foram: mata de galeria, que corresponde às formações florestais associadas aos cursos d'água; varjão, que inclui as fitofisionomias de varjão limpo e varjão sujo (campo sujo e campo limpo), em que o varjão sujo consiste de formação savânica caracterizada pela presença marcante de árvores agrupadas em pequenas elevações do terreno, conhecidas como murundus ou monchões, e o varjão limpo é uma fitofisionomia predominantemente herbácea de espécies gramíneo-lenhosas e árvores esparsas (Martins et al., 2006); ipucas, que são fragmentos naturais de florestas inundáveis inseridos dentro dos varjões; e cerrado, que abrange as fitofisionomias de cerrado típico e cerradão, descritas por Coutinho (1978). $\mathrm{O}$ uso antrópico correspondeu a áreas de agricultura e de pastagem, e o urbano incluiu a mancha urbana do Município de São Félix do Araguaia, MT. Os corpos 
d'água detectados referiam-se a rios, lagos e lagoas; e os bancos de areia, a acumulações de areias, aluviões e seixos nas margens dos rios.

A delimitação da área de estudo foi executada de forma automatizada, a partir de modelo digital de elevação "shuttle radar topographic mission" (SRTM), com resolução espacial de $30 \mathrm{~m}$, integrado e processado em sistema de informações geográficas. A metodologia utilizada nesse processo foi subdividida em quatro etapas: preenchimento de depressões ("fill sinks"), direção de fluxo ("flow direction"), fluxo acumulado ("flow accumulation") e delimitação de bacias ("watershed"), conforme Alves Sobrinho et al. (2010).

Como base de dados, utilizou-se imagem do sensor $\mathrm{TM} /$ Landsat, órbita 223, ponto 068 , de 5/9/2010, que envolveu as seguintes bandas espectrais: bandas $1(0,45-0,52 \mu \mathrm{m}) ; 2(0,50-0,60 \mu \mathrm{m}) ; 3(0,63-0,69 \mu \mathrm{m})$; $4(0,76-0,90 \mu \mathrm{m}) ; 5(1,55-1,75 \mu \mathrm{m})$ e $7(2,08-2,35 \mu \mathrm{m})$. Também foram utilizadas carta da cobertura vegetal do Bioma Cerrado SC-22-Z-C na escala 1:250.000 (Mapas de cobertura vegetal dos biomas brasileiros, 2006), imagem georreferenciada e ortorretificada do sensor ETM + Landsat, órbita/ponto 223/068, de 19/10/2000, obtida junto ao Global Land Cover Facility (2012), imagens ortorretificadas HRG SPOT 5 (modo SPOT Maps) MI 1767/1824 e imagens Ikonos disponíveis no programa Google Earth Pro.

Inicialmente, a imagem foi convertida para radiância, de acordo com Markham \& Barker (1987). Este procedimento foi realizado banda a banda (total de seis bandas) para a cena TM/Landsat-5. Em seguida, a imagem resultante foi transformada para o formato banda intercalada por pixel (BIP), que representa a maneira em que as múltiplas bandas da imagem digital são armazenadas. Os valores digitais de cada pixel foram armazenados sequencialmente para todas as bandas, e a correção atmosférica foi aplicada por meio do "fast line-of-sight atmospheric analysis of spectral hypercubes" (Flaash), que utiliza o código de transferência radiativa "moderate resolution transmittance” (ModTran) para a conversão das radiâncias em reflectâncias na superfície terrestre (Adler-Golden et al., 1999; Berk et al., 2002).

A correção geométrica foi realizada tendo como base a imagem ETM + Landsat-7, órbita/ponto 223/068, de 29/10/2000, georreferenciada e ortorretificada, cuja primeira etapa consistiu na identificação e no registro de 25 pontos de controle terrestre, nas duas cenas. A avaliação da qualidade dos pontos de controle foi feita com base no erro médio quadrático total (RMS), tendo-se considerado valor abaixo de 0,50 pixel adequado para o propósito do presente trabalho. A posição de cada pixel na imagem foi realocada para sua posição correta na imagem corrigida, mediante utilização da regressão polinomial de primeiro grau, em que os pixels foram reamostrados pelo método do vizinho mais próximo. Este método apenas atribuiu o valor do nível de cinza de determinado pixel da imagem reamostrada ao pixel da imagem original que estava mais próximo.

Esta reamostragem apresentou, como vantagens, rápido processamento e fácil implementação, além de não ter alterado os valores radiométricos da imagem original (Novo, 2010). Utilizou-se, como referência, o sistema de coordenadas geográficas (latitude e longitude) e, como datum, o elipsoide "World geodetic system 1984".

A imagem foi submetida a duas técnicas de matemática de bandas espectrais, o MNDWI e o NDVI. O MNDWI foi aplicado no processo de classificação para auxiliar na classificação dos corpos d'água e foi executado conforme Xu (2006), de acordo com a seguinte relação: $M N D W I=\left(R_{V E}-R_{I V M}\right) /\left(R_{V E}+R_{I V M}\right)$, em que $R_{V E}$ corresponde à reflectância no canal verde (banda 2 do sensor TM/Landsat), e $\mathrm{R}_{\mathrm{IVM}}$ corresponde à reflectância no canal do infravermelho médio (banda 5 do sensor TM/Landsat). O NDVI auxiliou na determinação das classes de vegetação ipucas, matas de galeria e cerrado, e foi aplicado conforme equação proposta por Rouse et al. (1973): NDVI $=\left(R_{I V P}-R_{V}\right) /$ $\left(\mathrm{R}_{\mathrm{IVP}}+\mathrm{R}_{\mathrm{V}}\right)$, em que $\mathrm{R}_{\mathrm{IVP}}$ corresponde à reflectância no canal do infravermelho próximo (banda 4 do sensor $\mathrm{TM}$ / Landsat), e $\mathrm{R}_{\mathrm{V}}$ corresponde à reflectância no canal vermelho (banda 3 do sensor TM/Landsat).

Em seguida, foi aplicado o processo de segmentação por crescimento de regiões (Baatz \& Schäpe, 2000), implementado no programa eCognition 8.2 (Trimble GeoSpatial, 2009). O processo de segmentação pode ser aplicado por três parâmetros definidos pelo usuário: escala, forma e compacidade. O parâmetro de escala determina a heterogeneidade máxima permitida para os objetos da imagem, e seu valor varia de acordo com o tamanho dos objetos resultantes; assim, um parâmetro de escala com valores maiores produz objetos maiores. O parâmetro de forma consiste na união dos parâmetros de compacidade e suavidade, em que o parâmetro 
compacidade é definido pela razão da área do objeto e o raio da circunferência abrangente, e o parâmetro de suavidade expressa o limite entre objetos, em que, quanto mais fractal, maior o índice de forma (Esch et al., 2008; Evans et al., 2010). Para a definição da melhor segmentação, foram avaliados - com base nas médias dos valores das seis bandas espectrais do sensor TM/Landsat - os seguintes parâmetros de escala: $250,200,150,100,50$ e 30 , seguido dos critérios de forma e compacidade definidos como 0,3 e 0,5 , respectivamente.

A etapa final no processo de caracterização de cobertura da terra foi a classificação orientada a objetos. Foram utilizadas, como dados de entrada, as imagens MNDWI, NDVI e todas as bandas multiespectrais TM/Landsat-5 (Figura 1). Utilizaram-se os algoritmos de atribuição de classes ("assign class") para o mapeamento de: corpos d'água, mata de galeria, ipucas, cerrado e banco de areia, tendo como base de domínio na imagem a aplicação dos descritores de média, área e relação de borda. Para o mapeamento das classes mata de galeria, ipucas e cerrado, foi necessário criar uma classe mãe, denominada vegetação, a partir dos valores médios gerados da imagem NDVI. No mapeamento das classes uso antrópico e varjão, foi utilizado o algoritmo "vizinho mais próximo", a partir do valor médio de todas as bandas espectrais. Para a classificação da área urbana, utilizou-se edição manual. Após a classificação, foi realizada uma reclassificação por meio da edição manual dos dados, para corrigir eventuais falhas do mapeamento das classes de uso e cobertura da terra.

Foram realizadas duas campanhas de campo para a validação do mapeamento. A primeira ocorreu em outubro de 2010 e a segunda em março de 2011. A quantidade mínima de pontos utilizados para validação foi definida conforme Jensen (1996): $\mathrm{N}=4 \mathrm{p} \times \mathrm{q} / \mathrm{E}^{2}$, em que $\mathrm{p}$ é o percentual de exatidão esperado; $q=1$ - p; e E é o erro permissível. Para obter o percentual de exatidão de $90 \%$ e erro admissível de $5 \%$, o número mínimo de pontos utilizados deveria ser igual a 144. No entanto, optou-se por escolher um número duas vezes maior que esse, ou seja, igual a 288 pontos, para aumentar a confiabilidade da validação. Destes, apenas 96 foram amostrados durante as campanhas de campo, e, para a validação dos demais pontos, foram utilizadas as imagens HRG SPOT 5 modo SPOTMaps (2,5 m de resolução espacial), de 2009 - e as imagens Ikonos disponíveis no GoogleEarth Pro, como fontes de validação.

Para a avaliação dos resultados das classificações, foram utilizados os coeficientes de exatidão global e Kappa, gerados a partir da matriz de confusão. A matriz de confusão compara, classe por classe, a relação entre os dados de referência conhecidos e os resultados

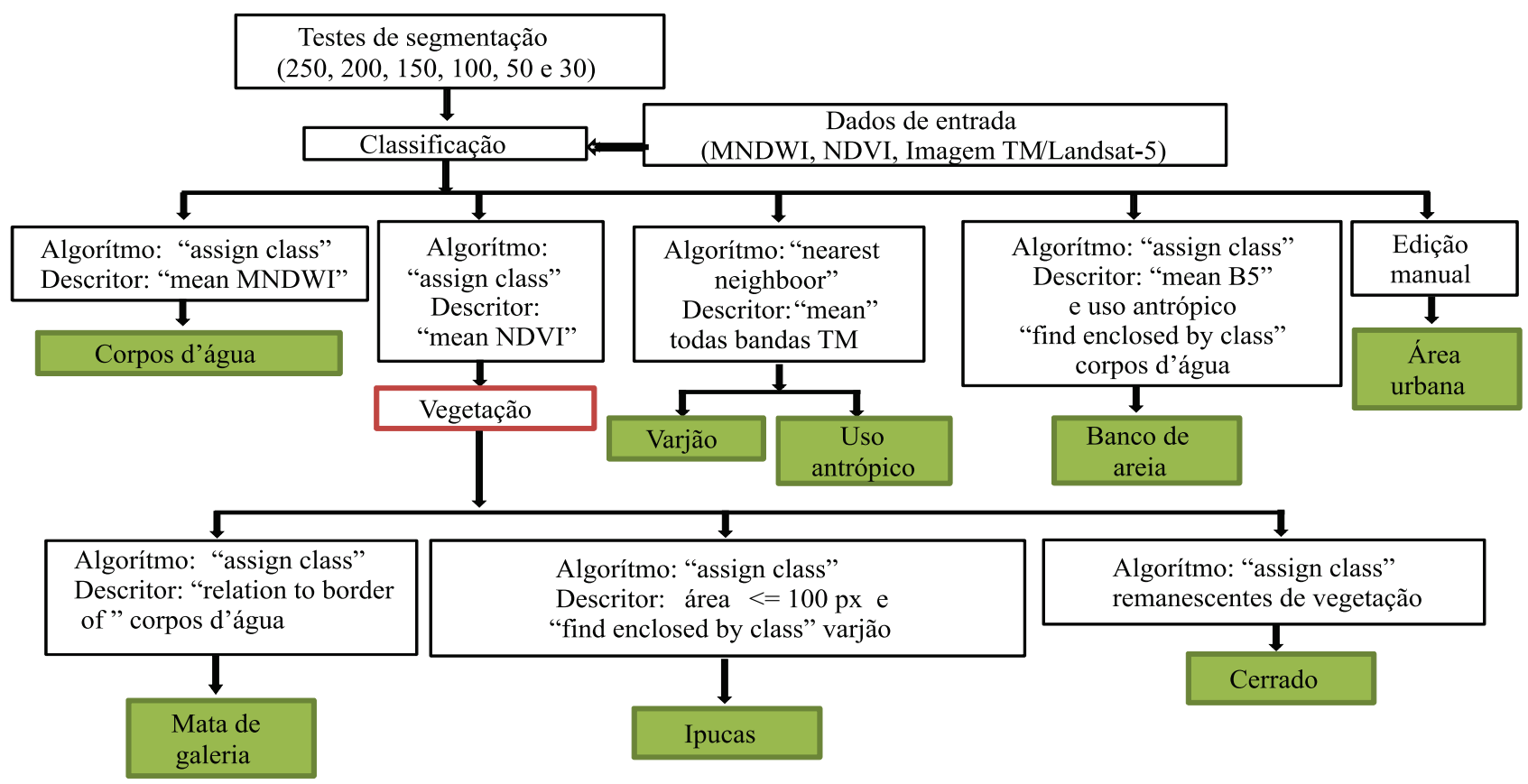

Figura 1. Fluxograma da sequência dos algoritmos e dos descritores utilizados no processo de classificação. 
correspondentes de uma classificação automatizada (Lillesand et al., 2004). Para conceituar os resultados obtidos pelo coeficiente Kappa, foram utilizados os intervalos pré-definidos por Landis \& Koch (1977).

\section{Resultados e Discussão}

A imagem obtida pelo índice MNDWI gerou valores entre os intervalos de $-1,09$ a 2,45, que possibilitaram o realce dos corpos d'água (Figura 2 A). Os intervalos do índice MNDWI que melhor definiram os corpos d'água foram relacionados diretamente aos valores mais positivos, e apresentaram variação de 0,48 a 2,06. Os valores do MNDWI entre 2,07 e 2,45 foram referentes a nuvens. Já os valores de 0,48 a $-1,09$, envolveram as demais feições sem realce em comparação ao índice MNDWI (área urbana, solo exposto, unidades fitofisionômicas e áreas de uso antrópico).

O índice NDVI apresentou valores entre -1 e 1, relativos à vegetação verde sadia. Esse índice possibilitou melhor realce das classes: matas de galeria, ipucas e cerrado. Os intervalos do índice NDVI que definiram essas classes foram de 0,44 a 0,63. A difícil distinção entre as áreas de varjão e as de uso antrópico deve-se a respostas espectrais similares obtidas nessas classes (Figura 2 B).
Com relação aos testes de segmentação da imagem, optou-se por selecionar o parâmetro de escala que apresentasse melhores diferenças visuais significativas no tamanho e na forma dos objetos obtidos, referentes às classes de uso e cobertura da terra. De acordo com Pratt et al. (2007), nenhum resultado de segmentação, mesmo que aprovado em testes quantitativos, será convincente se não satisfizer a análise visual, que é a fonte mais robusta e experiente para a avaliação das técnicas de segmentação.

Observou-se que, para os parâmetros de escala 250, 200 e 150, os resultados obtidos apresentaram maior agregação dos objetos. Isso pode ocasionar generalização entre as classes e resultar em erros no processo de classificação da imagem (Figura $3 \mathrm{~A}, \mathrm{~B}$ e C). Esses objetos foram considerados inapropriados para o processo de classificação digital da imagem.

Os parâmetros de escala 100 e 50 apresentaram bom desempenho, no processo de segmentação, para boa parte das classes de uso e cobertura da terra; no entanto, para a individualização dos objetos relacionados às ipucas, esses parâmetros não tiveram resultados consistentes (Figuras $3 \mathrm{D}$ e E). Já o parâmetro de escala 30, que gerou objetos menores, foi o escolhido para execução do processo de classificação orientada a objetos, por ter fornecido detalhamento mais preciso das classes de uso e cobertura da terra e permitido a

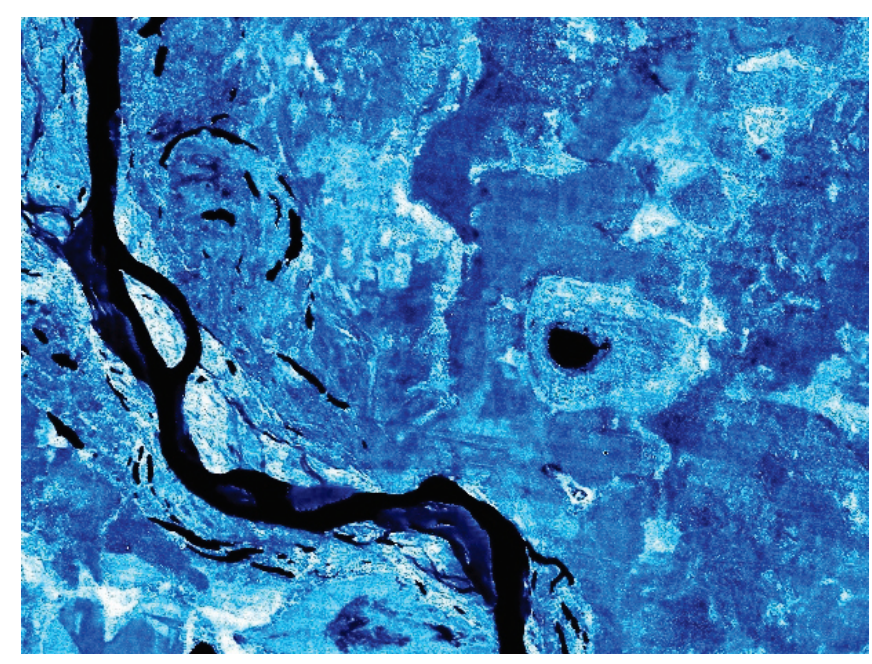

A

$-1,09$

2,45

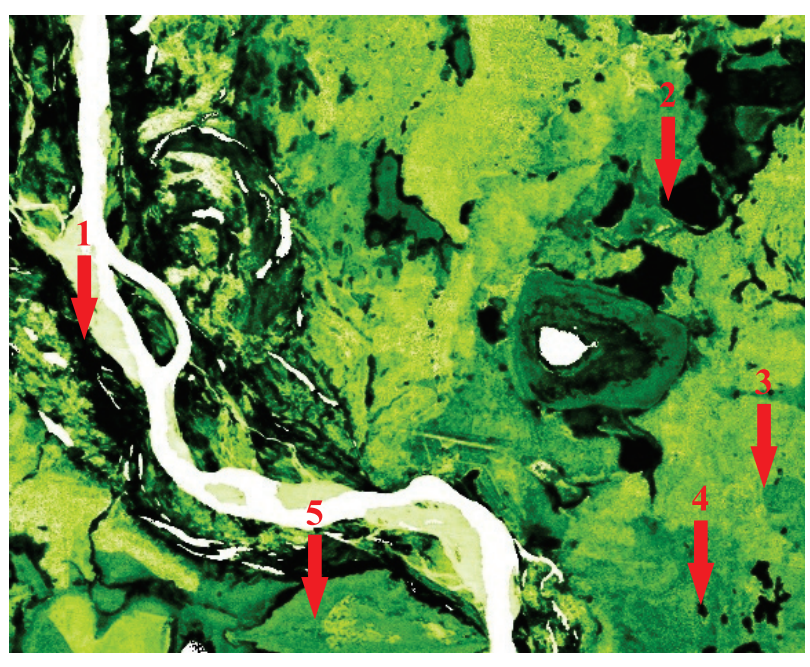

$-1$

Figura 2. Resultados obtidos pelo índice de água por diferença normalizada modificado (A) e detalhes das unidades de vegetação na imagem do índice de vegetação por diferença normalizada (B): 1, matas de galeria; 2, cerrado; 3, varjão; 4, ipucas; e 5, uso antrópico. 
obtenção de resultados de classificação mais refinados (Figura $3 \mathrm{~F}$ ).

Entre as classes de uso e cobertura, predominaram as de varjão (sujo e limpo), que cobriram $71,6 \%$ da área (138.984 ha), seguidas das classes mata de galeria com 9,5\% (18.383 ha), cerrado (típico e cerradão) com 7,9\% (15.296 ha), de uso antrópico (agricultura e pastagem) com 4\% (7.850 ha), ipucas (fragmentos naturais de florestas inundáveis) com 2,9\% (5.694 ha), corpos d'água com 2,8\% (5.426 ha), banco de areia com 1\% (1.898 ha) e área urbana com 0,3\% (523 ha) (Figura 4).

A maior extensão de varjões nessa região corrobora os dados de Martins et al. (2002), que utilizaram a classificação fitofisionômica e de uso antrópico para diagnosticar fragmentos florestais naturais em área pontual no Município de Lagoa da Confusão, TO. Estes autores verificaram que as áreas de varjão foram as que apresentaram as maiores contribuições na composição da paisagem.

As classes com menor confusão foram as de área urbana e de bancos de areia, e as que apresentaram maior confusão, as de varjão e uso antrópico (Tabela 1). As áreas de varjão obtiveram pixels classificados erroneamente para as classes cerrado, mata de galeria, ipucas e banco de areia, e o cerrado foi a classe que apresentou maior confusão com o varjão. Ainda assim, o varjão ainda foi a segunda classe com maior percentual de exatidão no mapeamento ( $92 \%)$. A classe de uso antrópico obteve maior confusão com as classes banco de areia, principalmente, e varjão, cerrado e corpos d'água. A classe de área urbana foi a que mostrou maior percentagem de acerto na classificação, com $100 \%$ de exatidão.

De um total de 288 pontos amostrados na validação, 240 foram mapeados corretamente, o que resultou em índice de exatidão global de 83,3\%. O valor do coeficiente de concordância Kappa foi de 0,72, considerado muito bom, segundo Landis \& Koch(1977). Um fator que contribuiu para a diminuição da exatidão global foi a transição gradual e complexa das áreas de cerrado e varjão para as fitofisionomias adjacentes. De acordo com Sano et al. (2010), este bioma apresenta forte sazonalidade e alta heterogeneidade em termos de biodiversidade e fitofisionomia, o que dificulta o processo de classificação digital da imagem.

Estes resultados evidenciam o potencial da utilização da classificação orientada a objetos em mapeamentos de uso e cobertura da terra, na região do Médio Araguaia. Luz et al. (2010), ao realizar mapeamento de uso da terra com imagens SPOT-5, em área no interior do Estado do Paraná, por meio das técnicas de segmentação multiresolução e classificação orientada a objetos, obtiveram resultados semelhantes. Desclée et al. (2006) analisaram a utilização da classificação orientada a objetos para detecção de alterações da cobertura florestal, e verificaram que a precisão na detecção de mudanças realizadas por este método foi maior que a obtida com base em pixels, independentemente da fonte de dados de validação. A mesma observação foi feita por Fernandes et al. (2011) que, ao comparar, por
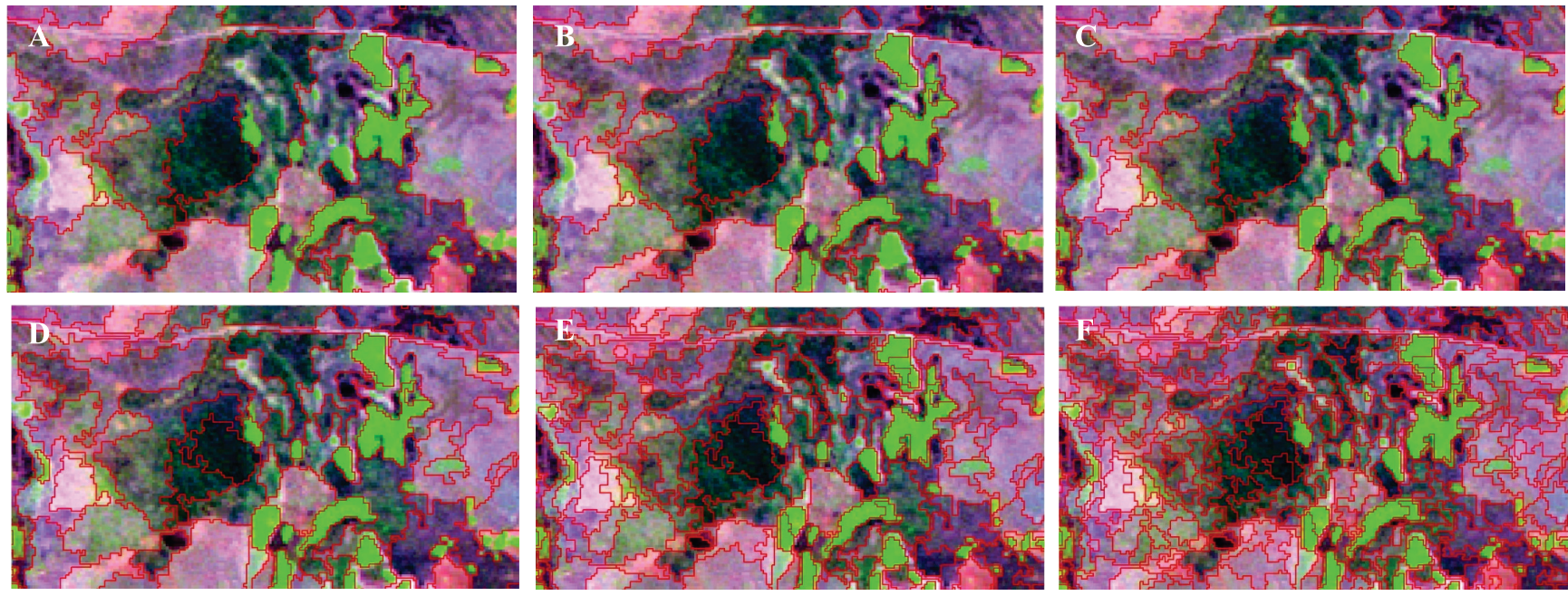

Figura 3. Resultados obtidos de acordo com os parâmetros de escala aplicados nos testes de segmentação: A, 250; B, 200; C, 150; D, 100; E, 50; e F, 30 . 
máxima verossimilhança, a classificação orientada a objetos com o classificador pixel-a-pixel, no mapeamento de áreas degradadas no Estado do Mato
Grosso, observaram que a classificação orientada a objetos foi a que apresentou resultados finais mais promissores.

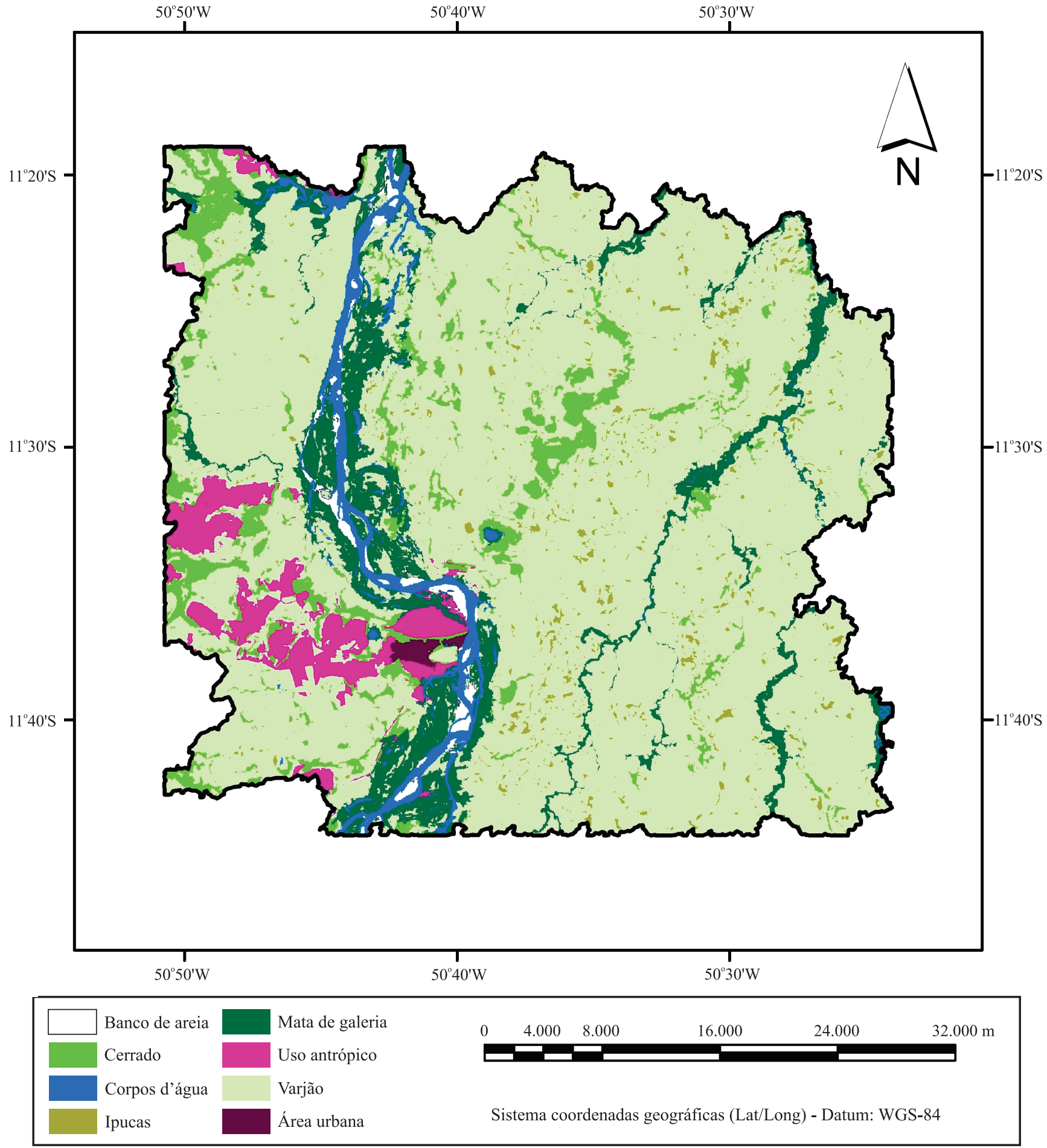

Figura 4. Mapa de classes de uso e cobertura da terra resultante do processo de classificação orientada a objetos da planície de inundação do Médio Rio Araguaia, em 5/9/2010. 
Tabela 1. Matriz de confusão em relação à separabilidade das amostras, com base nos resultados da classificação orientada a objetos para as diferentes classes consideradas no mapeamento de cobertura da terra, na região do Médio Araguaia.

\begin{tabular}{|c|c|c|c|c|c|c|c|c|c|}
\hline Classes & Uso antrópico & Varjão & Cerrado & Ipucas & Mata de galeria & Corpos d'água & Banco de areia & Área urbana & Total \\
\hline Uso antrópico & 13 & 2 & 0 & 0 & 0 & 0 & 0 & 0 & 15 \\
\hline Varjão & 5 & 159 & 5 & 1 & 6 & 2 & 1 & 0 & 179 \\
\hline Cerrado & 1 & 5 & 17 & 1 & 0 & 0 & 0 & 0 & 24 \\
\hline Ipucas & 0 & 1 & 0 & 15 & 0 & 0 & 0 & 0 & 16 \\
\hline Mata de galeria & 0 & 4 & 11 & 0 & 20 & 1 & 0 & 0 & 36 \\
\hline Corpos d'água & 0 & 0 & 0 & 0 & 1 & 9 & 0 & 0 & 10 \\
\hline Banco de areia & 0 & 1 & 0 & 0 & 0 & 0 & 4 & 0 & 5 \\
\hline Área urbana & 0 & 0 & 0 & 0 & 0 & 0 & 0 & 3 & 3 \\
\hline Total & 19 & 172 & 33 & 17 & 27 & 12 & 5 & 3 & 288 \\
\hline Acerto (\%) & 68 & 92 & 52 & 88 & 74 & 75 & 80 & 100 & \\
\hline
\end{tabular}

A classificação orientada a objetos tem maior aplicação em imagens de alta resolução espacial; no entanto, os resultados obtidos no presente trabalho são satisfatórios para aplicação em imagens de média resolução espacial, como as imagens TM/Landsat. Portanto, a classificação orientada pode ser uma excelente ferramenta para tomadas de decisão em estudos relacionados ao manejo de paisagens, bem como delimitação de habitats específicos, como no caso da classificação e da delimitação de ipucas. Estas fitofisionomias apresentam resposta espectral muito similar às dos varjões e das matas de galeria, o que dificultaria a sua classificação por outro método de classificação convencional.

Portanto, a classificação orientada a objetos apresenta muitas potencialidades, entre as quais destacam-se a segmentação das imagens em mais de uma escala, a classificação com mais de um conjunto de parâmetros, e a possibilidade de exploração de atributos espectrais, de textura, geométricos e topológicos, para a classificação de segmentos. A classificação orientada também possibilita a elaboração de regras e processos complexos de classificação, bem como a análise de imagens de forma flexível, o que permite a emulação do raciocínio humano de interpretação em imagens de sensoriamento remoto (Blaschke, 2010). Além disso, ela apresenta contribuições significativas para o monitoramento ambiental, com grande potencial para melhorar a precisão de trabalhos relacionados à classificação de uso e cobertura da terra (Platt \& Rapoza, 2008).

A classificação orientada a objetos, apesar de ainda pouco difundida, é uma abordagem que tomará frente a inúmeros estudos relacionados à verificação e à disposição dos recursos naturais, quanto à sua distribuição espacial. Resultados melhores podem ser obtidos com esta metodologia, em estudos futuros, com o uso de dados ópticos de baixa, média e alta resolução espacial, integrados com dados radar, para testar metodologias existentes (Evans et al., 2010). Dados provenientes de multisensores poderiam fornecer maiores informações e definir melhor as classes mapeadas, como varjão e uso antrópico, que apresentaram certa confusão nos resultados referentes ao índice NDVI e foram mapeadas com uso do algoritmo "nearest neighbor".

\section{Conclusões}

1. As matemáticas de bandas espectrais aplicadas na imagem $\mathrm{TM} /$ Landsat, associadas ao processo de classificação em conjunto, com segmentação em escala refinada, apresentam resultados promissores no delineamento das classes de uso e cobertura da terra, na região do Médio Araguaia.

2. O método de classificação orientada a objetos, pode ser reaplicado em outras áreas com características semelhantes às da região do Médio Araguaia, para avaliação da disposição dos recursos naturais quanto a sua distribuição espacial.

\section{Agradecimentos}

Ao Instituto Nacional de Ciência e Tecnologia em Áreas Úmidas, Programa Institutos Nacionais de Ciência e Tecnologia do Conselho Nacional de Desenvolvimento Científico e Tecnológico, pelo apoio financeiro. 


\section{Referências}

ADLER-GOLDEN, S.M.; MATTHEW, M.W.; BERNSTEIN, L.S.; LEVINE, R.Y.; BERK, A.; RICHTSMEIER, S.C.; ACHARYA, P.K.; ANDERSON, G.P.; FELDE, G.; GARDNER, J.; HOKE, M.; JEONG, L.S.; PUKALL, B.; MELLO, J.; RATKOWSKI, A.; BURKE, H.-H. Atmospheric correction for short-wave spectral imagery based on MODTRAN4. Proceedings of the Society of Photo-Optical Instrumentation Engineers, v.3753, p.61-69, 1999.

ALVES, C.D.; PEREIRA, M.N.; FLORENZANO, T.Z.; SOUZA, Í. de M. Análise orientada a objeto no mapeamento de áreas urbanas com imagens Landsat. Boletim de Ciências Geodésicas, v.15, p.120-141, 2009.

ALVES SOBRINHO, T.; OLIVEIRA, P.T.S.; RODRIGUEIS, D.B.B.; AYRES, F.M. Delimitação automática de bacias hidrográficas utilizando dados SRTM. Revista Engenharia Agrícola, v.30, p.46-57, 2010.

ANTUNES, A.F.B. Classificação de ambiente ciliar baseada em orientação a objeto em imagens de alta resolução espacial. 2003. 147p. Tese (Doutorado) - Universidade Federal do Paraná, Curitiba.

BAATZ, M.; SCHÄPE, A. Multiresolution segmentation: an optimization approach for high quality multi-scale image segmentation. Journal of Photogrammetry and Remote Sensing, v.58, p.12-23, 2000.

BERK, A.; ADLER-GOLDEN, S.M.; RATKOWSKI, A.J.; FELDE, G.W.; ANDERSON, G.P.; HOKE, M.L.; COOLEY, T.; CHETWYND, J.H.; GARDNER, J.A.; MATTHEW, M.W.; BERNSTEIN, L.S.; ACHARYA, P.K.; MILLER, D.; LEWIS, P. Exploiting MODTRAN radiation transport for atmospheric correction: the FLAASH algorithm. In: INTERNATIONAL CONFERENCE ON INFORMATION FUSION, 5., 2002, Annapolis. Proceedings. Annapolis: ISIF, 2002. v.2, p.798-803.

BLASCHKE, T. Object-based image analysis for remote sensing. Journal of Photogrammetry and Remote Sensing, v.65, p.2-16, 2010 .

COUTINHO, L.M. O conceito de cerrado. Revista Brasileira de Botânica, v.1, p.17-23, 1978.

DESCLÉE, B.; BOGAERT, P.; DEFOURNY, P. Forest change detection by statistical object-based method. Remote Sensing of Environment, v.102, p.1-11, 2006.

DUVEILlER, G.; DEFOURNY, P.; DESCLÉE, B.; MAYAUX, P. Deforestation in Central Africa: estimates at regional, national and landscape levels by advanced processing of systematically-distributed Landsat extracts. Remote Sensing of Environment, v.112, p.1969-1981, 2008.

ESCH, T.; THIEL, M.; BOCK, M.; ROTH, A.; DECH, S. Improvement of image segmentation accuracy based on multiscale optimization procedure. IEEE Geoscience Remote Sensing Letters, v.5, p.463-467, 2008.

EVANS, T.L.; COSTA, M.; TELMER, K.; SILVA, T.S.F. Using ALOS/PALSAR and RADARSAT-2 to map land cover and seasonal inundation in the Brazilian Pantanal. IEEE Journal of
Selected Topics in Applied Earth Observations and Remote Sensing, v.3, p.560-575, 2010.

FERNANDES, R.R.; NUNES, G.M.; DRESCHER, R. Monitoramento de propriedades rurais através de dados multisensores em nível orbital. Ambiência, v.7, p.75-87, 2011.

GLOBAL LAND COVER FACILITY. Global Land Cover Facility [home page]. Available at: <http://glcf.umiacs.umd.edu/ index.shtml>. Accessed on: 17 Oct. 2012.

JENSEN, J.R. Introductory digital image processing: a remote sensing perspective. 2.ed. Upper Saddle River: Prentice Hall, 1996. $316 \mathrm{p}$.

LANDIS, J.R.; KOCH, G.G. The measurement of observer agreement for categorical data. Biometrics, v.33, p.159-174, 1977.

LILLESAND, T.M.; KIEFER, R.W.; CHIPMAN, J.W. Remote sensing and image interpretation. 5.ed. Madison: Wiley, 2004. $763 \mathrm{p}$.

LUZ, N.B. da; ANTUNES, A.F.B.; TAVARES JÚNIOR, J.B. Segmentação multirresolução e classificação orientada a objetos aplicados a imagens Spot-5 para o mapeamento do uso da terra. Floresta, v.40, p.429-446, 2010.

MAPAS de cobertura vegetal dos biomas brasileiros. Brasília: Ministério do Meio Ambiente, 2006. Disponível em: $<$ http://mapas. mma.gov.br/mapas/aplic/probio/datadownload.htm/>. Acesso em: 13 mar. 2011.

MARKHAM, B.L.; BARKER, J.L. Thematic mapper bandpass solar exoatmospheric irradiances. International Journal of Remote Sensing, v.8, p.517-523, 1987.

MARTINS, A.K.E.; SCHAEFER, C.E.G.R.; SILVA, E.; SOARES, V.P.; CORRÊA, G.R.; MENDONÇA, B.A.F. Relações solo-geoambiente em áreas de ocorrências de ipucas na planície do Médio Araguaia - Estado do Tocantins. Revista Árvore, v.30, p.297-310, 2006.

MARTINS, Y.C.M.; SOARES, V.P.; SILVA, E.; BRITES, R.S. Diagnóstico ambiental no contexto da paisagem de fragmentos florestais naturais "ipucas" no Município de Lagoa da Confusão, Tocantins. Revista Árvore, v.26, p.299-309, 2002.

MCFEETERS, S. K. The use of normalized difference water index (NDWI) in the delineation of open water features. International Journal of Remote Sensing, v.17, p.1425-1431, 1996.

MILESKI, E. Aspecto da vegetação e do ecossistema da Ilha do Bananal: mapa fitoecológico e indicadores de pressão antrópica. Brasília: Presidência da República, 1994. 104p.

NOVO, E.M.L. de M. Sensoriamento remoto: princípios e aplicações. 4.ed. São Paulo: Edgard Blücher, 2010. 387p.

PINHO, C.M.D. de; FONSECA, L.M.G.; KORTING, T.S.; ALMEIDA, C.M. de; KUX, H.J.H. Land-cover classification of an intra-urban environment using high-resolution images and object-based image analysis. International Journal of Remote Sensing, v.33, p.5973-5995, 2012.

PLATT, R.V.; RAPOZA, L. An evaluation of an object-oriented paradigm for land use/land cover classification. The Professional Geographer, v.60, p.87-100, 2008. 
PRATT, W.K. Digital image processing: PIKS scientific inside. 4.ed. New York: J. Wiley, 2007. 786p.

ROUSE, J.W.; HAAS, R.H.; SCHELL, J.A.; DEERING, D.W. Monitoring vegetation systems in the Great Plains with ERTS. In: EARTH RESOURCES TECHNOLOGY SATELLITE-1 SYMPOSIUM, 3., 1973, Washington. Proceedings. Washington: National Aeronautics and Space Administration, 1973. p.309-317.

SANO, E.E.; ROSA, R.; BRITO, J.L.S.; FERREIRA, L.G. Land cover mapping of the tropical savanna region in Brazil. Environmental Monitoring and Assessment, v.166, p.113-124, 2010.

TRIMBLE GEOSPATIAL. Definiens eCognition developer. Version 8. Munich: Trimble GeoSpatial, 2009.
WANG, Z.X.; LIU, C.; HUETE, A. From AVHRR-NDVI to MODIS-EVI: advances in vegetation index research. Acta Ecologica Sinica, v.23, p.979-987, 2003.

$\mathrm{XU}, \mathrm{H}$. Modification of normalised difference water index (NDWI) to enhance open water features in remotely sensed imagery. International Journal of Remote Sensing, v.27, p.3025-3033, 2006.

YAN, G.; MAS, J.-F.; MAATHUIS, B.H.P.; XIANGMIN, Z.;VAN DIJK, P.M. Comparison of pixel-based and object-oriented image classification approaches - a case study in a coal fire area, Wuda, Inner Mongolia, China. International Journal of Remote Sensing, v.27, p.4039-4055, 2006.

Recebido em 29 de julho de 2011 e aprovado em 5 de agosto de 2012 